\title{
Fatal complication of infective endocarditis: Radiological diagnosis of aortomitral curtain pseudoaneurysm
}

\author{
Safiye Sanem Dereli Bulut \\ Radiology Department, TC Health Sciences University, Umraniye Research and Training Hospital, Istanbul, Turkey
}

Received: 2020-06-18.

Accepted: 2020-07-16

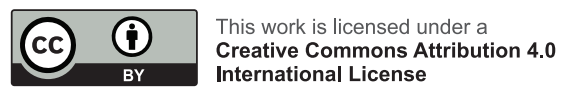

J Clin Med Kaz 2020; 5(59):51-53

Corresponding author:

Safiye Sanem Dereli Bulut.

E-mail: ssanembulut@gmail.com

\section{Abstract}

Infective endocarditis is the infection of the endocardium. The pathologic hallmarks of infective endocarditis are vegetations. They appear as irregular, mobile or fixed masses that usually attach to the endocardium on the low-pressure side of a valve, chordae tendinae or ascending aorta.

Duke criteria are clinically relevant for the diagnosis of infective endocarditis. In addition to clinical and laboratory findings with these criteria, the diagnosis can be made with high accuracy with imaging findings. Imaging also plays a decisive role in pre-operative evaluation, during heart valve operations and in the evaluation of often severe complications to the disease.

We present a 75-year-old male patient who was diagnosed with aortomitral curtain pseudoaneurysm due to infective endocarditis after clinical, laboratory and radiological evaluation in the light of literature review.

Key words: aneurysm, false, endocarditis, magnetic resonance Imaging

\section{Introduction}

The definition of infective endocarditis (IE) is the infection of the endocardium. It commonly affects the leaflets of valves and chordae tendineae. Especially most commonly affects prosthetic valves and implanted devices. There is an increased risk with intravenous drug users. It is approximately two times more common in men [1].

Although the rates of medical diagnosis and treatment have increased, the rates of IE related mortality are around $20 \%$ even in developed countries [1].

Echocardiography is the primary imaging modality of cardiac infections. Computed Tomography (CT) and Magnetic Resonance Imaging (MRI) can detect valvular vegetation features in more detail than echocardiography $[2,3]$.

Differential diagnosis may include papillary fibroelastoma, myxoma, lipoma-like tumors, or pathologies such as thrombus, valvular strands. Knowing the imaging findings of the identified pathologies provides a great ease of separation [1].
We present a 75-year-old male patient who was diagnosed with aortomitral curtain pseudoaneurysm due to infective endocarditis after clinical, laboratory and radiological evaluation. In doing so, we discussed the patient in the form of a literature review.

\section{Case-presentation}

A 75-year-old male patient with a history of spondylodiscitis and fifteen years ago prosthetic valve operation due to aortic valve insufficiency, applied to the hospital. In two sets of blood cultures, methicillinsensitive Staphylococcus aureus (MSSA) was isolated.

Lumbar spine MRI (1.5 Tesla, GE Healthcare Optima MR 450) showed spondylodiscitis, medullary edema signal, the left paravertebral area had a soft tissue component affecting the psoas muscle at L2L3 level. Tissue biopsy from this localization was the result of culture microorganism methicillin-sensitive catalase negative Staphylococcus aureus (MSSA). The susceptibility of isolate to antibiotics was determined using disk diffusion method according to CLSI (Clinical and Laboratory Standards Institute) guidelines. The strain 
was found to be sensitive to ampicillin, amoxicillin, ceftriaxone, nitrofurantoin, trimethoprim/sulfomethoksozol, vancomycin and resistant to erythromycin, clindamycin, and gentamicin. The $\beta$-lactam antibiotic treatment was planned orally for 10 days followed by parenteral treatment for 20 days.

Two months later after treatment, the patient was admitted to the hospital due to progressive dyspnea. The patient was interned due to poor general condition. Laboratory tests showed leukocytosis. Chest X-ray examination showed diffuse bilateral pulmonary edema.

His physical examination revealed a newly developed murmur. Transthoracic echocardiography (TTE) was planned for suspicion of infective endocarditis. TTE showed $60 \%$ left ventricular ejection fraction with new, moderate mitral regurgitation and probable vegetation on the anterior mitral leaflet (Figure 1).

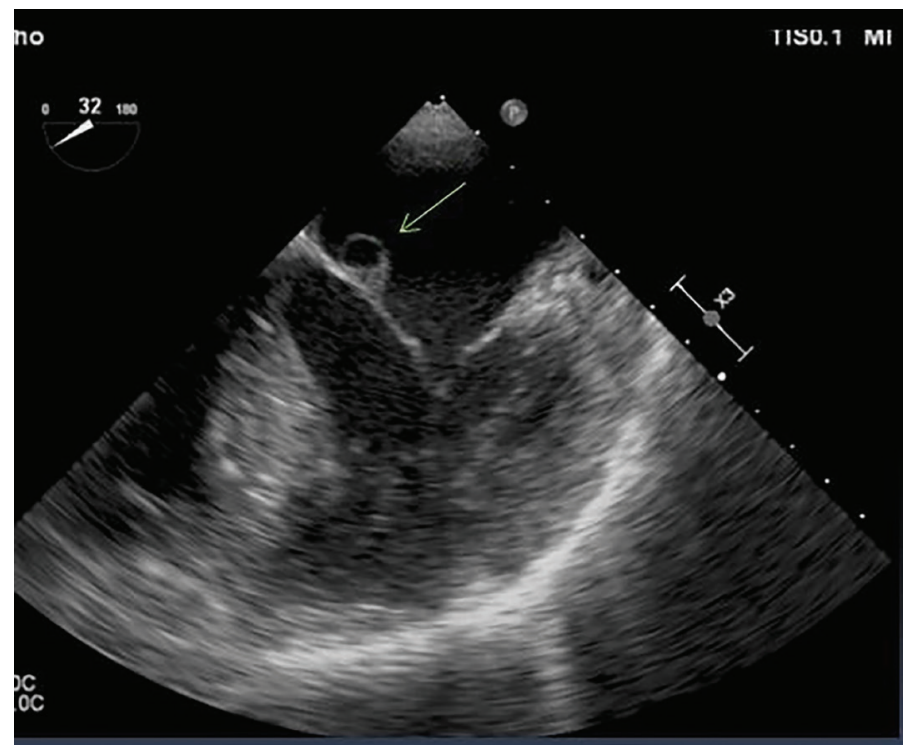

Figure 1 - A 75 year old man patient with progressive dyspnea, has history of spondilodiscitis and newly developed murmur: TTE shows that moderate mitral regurgitation and probable vegetation on the anterior mitral leaflet (straight arrow).

Aneurysmatic expansion causing turbulent flow at the mitral valve level was noted. ECG-gated Computed tomography angiography (CTA) was performed (GE Healthcare Optima CT660, 128 slices) to clarify the situation. During diastole, there was an outpouching of the anterior mitral leaflet at the aortomitral curtain area (Figure 2a,b). Subaortic abscess was considered in the differential diagnosis.

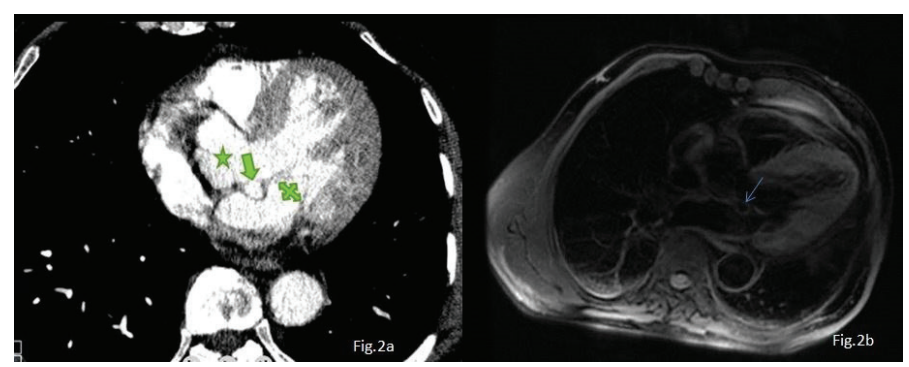

Figure 1 - A 75 year old man patient with progressive dyspnea, has history of spondilodiscitis and newly developed murmur; CTA findings during diastole: there was an outpouching of the anterior mitral leaflet at the aortomitral curtain area (straight arrow). Aortic cuspis level is marked with a green star, mitral valve level is marked with cross arrows (2a). Triple IR T2-weighted image MRI sequence shows aortic valve (av), mitral valve (mv), left ventricle (LV) and right ventricle (RV). The 3-chamber image shows outpouching in the aortomitral curtion (thin green short arrow) (2b).
For further investigation, cardiac MRI was planned to evaluate the lesion location and to evaluate valve functions. Cardiac MRI (1,5 Tesla, GE Healthcare Optima MR 450) demonstrated mitral-aortic intervalvular fibrosa pseudo aneurysm. The turbulence flow at this location was also revealed with cardiac MRI.

A serious complications of aortic valve infective endocarditis and the developement of pseudoaneurysm of the aortomitral curtain was demonstrated in this case.

\section{Discussion}

Streptococcus and Staphylococcus species consist $80 \%$ of etiology in IE cases. The proportions of these microorganisms vary depending on the presence of artificial or natural valves, the source of the infection, the age of the patient and other accompanying diseases. In recent years, Staphylococci have been the most common agents in many IE species $[1,2]$.

In a prospective cohort International Collaboration on Endocarditis (ICE) study involving 2781 adult IE patients from 28 countries, $\mathrm{S}$. aureus was the most common factor in all IE species [1].

Duke criteria with $80 \%$ sensitivity and specificity, are clinically relevant for the diagnosis of infective endocarditis. In addition to clinical and laboratory findings with these criteria, the diagnosis can be made with high accuracy with imaging findings $[1,2]$. Although it provides support to the diagnosis of IE by clinical findings in clinical practice, the criteria are less sensitive in patients with negative blood culture or small number of blood cultures, or those with prosthetic valves or right heart diseases.

According to Duke criteria, imaging findings are among the major criteria in the diagnosis of IE [1]. Echocardiography is the first-line modality for the diagnosis of IE [1-3]. Imaging also plays a decisive role in pre-operative evaluation, during heart valve operations and in the assessment of the often severe complications [2]. The sensitivity for the diagnosis of vegetation is $75 \%$ for TTE and $85-90 \%$ for transesophageal echocardiography (TEE) $[1,3]$.

Echocardiography is useful in both situations, allowing a detailed assessment of valvular lesions, a quantification of valve regurgitation, and the evaluation of the hemodynamic tolerance of the regurgitation (cardiac output, pulmonary arterial pressures, left and right ventricular function) [4]. However, it is the most important disadvantages of being user dependent and insufficient in the evaluation of right heart [4].

In cases where TTE is not sufficient, cross-sectional imaging modalities can play an important role. As the major Duke criteria, CT findings in the diagnosis of IE were included in 2015. According to the IE guideline 2015, the detection of paravalvular lesion by CT was a major criterion $[3,4]$.

Cardiac-gated CTA can also demonstrate valve tissue destruction, and perivalvular extension with pseudoaneurysm or fistula formation [3]. However, CT may miss the small vegetations $[3,4]$.

In patients with infective endocarditis; in a study comparing CT and TEE with intraoperative findings for the presence of perivalvular abscess and pseudoaneurysm, the sensitivity for CT and TEE was $100 \%$ and $89 \%$, respectively. The specificity was $100 \%$ for both examinations [4]. TEE is superior in detecting small vegetations and valve perforations. However, CT seems to have an advantage in patients with more extensive calcification of the valves [4]. 
Valvular vegetations can be detected in cardiac MRI. The imaging findings of vegetations vary depending on the type of imaging sequence obtained [5,6]. More accurate information can be obtained with $\mathrm{CT}$ and MRI in detecting perivalvular extension of abscess or pseudoaneurysm compared to TEE. Cardiac MR imaging also allows quantification of regurgitation fraction [5-7].

Direct extension and tissue de $\neg$ struction by IE include annular or aortic abscess formation, aortic aneurysm or pseudoaneurysm, and fistula formation. The aortic and mitral valves are the valves more commonly in need of replacement or repair due to tissue destruction, which causes rapid heart failure $[6,7]$.

The mitral-aortic intervalvular fibrosa is the junctional tissue between the elements of the aortic and mitral valves. Anatomically, the perforation or defect in the mitral-aortic intervalvular fibrosa is located above the plane of the mitral anulus and below that of the aortic anulus [6,7].

Mitral-aortic intervalvular fibrosa infection occurs most commonly in patients with aortic valve infective endocarditis $[6,7]$. Infection of the aortic valve causes a regurgitant flow $[6,7]$.

Preoperative diagnosis is important in these cases. The differential diagnosis is the ruptured aneurysm of the Valsalva sinus and the perforation of the anterior mitral leaflet [5-7].

Valve tissue destruction, regurgitation, and peri $\neg$ valvular extension with pseudoaneurysm or fistula formation can be characterized in detail at cardiac MR imaging and cardiac CT angiography [5-7].

Cardiac MR imaging also allows quantification of regurgitation fraction with multiplanar delinea $\neg$ tion. Regurgitation jets demonstrate dephasing artifact at cine steadystate free precession (SSFP) imaging, and to-and-fro flow in pseudoaneurysms can be visualized throughout the cardiac cycle [5-7].

Perivalvular infection spread (abscess, pseudoaneurysms, valvular dissociation with fistulas and paravalvular leakage) is the second most common indication for early surgery in IE. Abscesses are collections of irregular shaped perivalvular fluids that do not come into contact with the blood pool in the heart chambers. In CTA examination, abscesses are seen in perivalvular regions as peripheral contrast enhanced hypodense fluid collections [4-7]. Pseudoaneurysms are irregularly shaped infected perivalvular cavities that communicate with the intracardiac blood pool and cause a pulsatile color flow in Doppler Echocardiography [4-7]. In dynamic cine CTA examination, filling with contrast medium is observed as a complex pulsatile perivalvular lesion areas [47].

\section{References}

1. Murillo H, Restrepo CS, Marmol-Velez JA, et al. Infectious Diseases of the Heart: Pathophysiology, Clinical and Imaging Overview. RadioGraphics. 2016; 36(4):963-83. https://doi.org/10.1148/rg.2016150225

2. Bruun NE,Habib G,Thuny F,Sogaard P; Cardiac imaging in infectious endocarditis. European Heart Journal. 2014; 35, 624-32 https:// doi.org/10.1093/eurheartj/eht274

3. Entrikin DW, Gupta P, Kon ND, Carr JJ. Imaging of infective endocarditis with cardiac CT angiography. J Cardiovasc Comput Tomogr. 2012; 6(6):399-405. https://doi.org/10.1016/j.jcct.2012.10.001

4. Feuchtner GM, Stolzmann P, Dichtl W, et al. Multislice Computed Tomography in Infective Endocarditis. Comparison With Transesophageal Echocardiography and Intraoperative Findings. J Am Coll Cardiol. 2009; 53(5):436-44. https://doi.org/10.1016/j. jacc.2008.01.077

5. Dursun M, Yilmaz S, Yilmaz E, et al. The utility of cardiac MRI in diagnosis of infective endocarditis: Preliminary results. Diagnostic Interv Radiol. 2015; 21(1):28-33. https://doi.org/10.5152/dir.2014.14239

6. Ryu S, Kim HJ, Kim SM, Park S, Choe YH. Detection of perivalvular abscess with late Gadolinium-Enhanced MR Imaging in a patient with Infective Endocarditis. Investig Magn Reson Imaging. 2016; (3):75-79. https://doi.org/10.13104/imri.2016.20.1.75

7. Bricker AO, Avutu B, Mohammed TLH, Williamson EE,et al. Valsalva Sinus Aneurysms: Findings at CT and MR Imaging. RadioGraphics. 2010; 30:99-110. https://doi.org/10.1148/rg.301095719 